NOTICE: this is the author's version of a work that was accepted for publication in Bone. Changes resulting from the publishing process, such as peer review, editing, corrections, structural formatting, and other quality control mechanisms may not be reflected in this document. Changes may have been made to this work since it was submitted for publication. A definitive version was subsequently published in Bone, Vol. 50, no.6 (2012).

DOI:10.1016/j.bone.2012.03.002 


\title{
Failure strength of human vertebrae: prediction using bone mineral density measured by DXA and bone volume by micro-CT
}

\author{
Egon Perilli ${ }^{1,2}$ \\ Andrew M Briggs ${ }^{3,4}$ \\ Susan Kantor ${ }^{4}$ \\ John Codrington ${ }^{1,5}$ \\ John D Wark ${ }^{4}$ \\ Ian H Parkinson ${ }^{1,2}$ \\ Nicola L Fazzalari ${ }^{1,2}$
}

${ }^{1}$ Bone and Joint Research Laboratory, SA Pathology and Hanson Institute, Adelaide, South Australia, Australia

${ }^{2}$ Discipline of Anatomy and Pathology, The University of Adelaide, Adelaide, South Australia

${ }^{3}$ Curtin Health Innovation Research Institute, Curtin University, Perth, Western Australia

${ }^{4}$ Department of Medicine, University of Melbourne, and Bone and Mineral Service, Royal Melbourne Hospital, Melbourne, Victoria, Australia

${ }^{5}$ School of Mechanical Engineering, The University of Adelaide, Adelaide, South Australia, Australia

\section{Corresponding Author:}

Egon Perilli, 
Bone and Joint Research Laboratory, SA Pathology and Hanson Institute, Frome Rd 5000 SA, Adelaide, South Australia, Australia. Tel: +618 82223269, Fax: +61882223293.

Email: egon.perilli@adelaide.edu.au

\section{$\underline{\text { Abstract }}$}

Significant relationships exist between areal bone mineral density (BMD) derived from dual energy X-ray absorptiometry (DXA) and bone strength. However, the predictive validity of BMD for osteoporotic vertebral fractures remains suboptimal. The diagnostic sensitivity of DXA in the lumbar spine may be improved by assessing BMD from lateral projection scans, as these might better approximate the objective of measuring the trabecular-rich bone in the vertebral body, compared to the commonly-used posterioranterior (PA) projections. Nowadays, X-ray micro-computed tomography $(\mu \mathrm{CT})$ allows non-destructive three-dimensional structural characterization of entire bone segments at high resolution. In this study, human lumbar cadaver spines were examined ex situ by DXA in lateral and PA projections, as well as by $\mu \mathrm{CT}$, with the aims (1) to investigate the ability of bone quantity measurements obtained by DXA in the lateral projection and in the PA projection, to predict variations in bone quantity measurements obtained by $\mu \mathrm{CT}$, (2) to assess their respective capabilities to predict whole vertebral body strength, determined experimentally.

Human cadaver spines were scanned by DXA in PA projections and lateral projections. Bone mineral content (BMC) and BMD for L2 and L3 vertebrae were determined. The L2 and L3 vertebrae were then dissected and entirely scanned by $\mu \mathrm{CT}$. Total bone volume $\left(\mathrm{BV}_{\text {tot }}=\right.$ cortical + trabecular $)$, trabecular bone volume $(\mathrm{BV})$, and trabecular bone 
volume fraction (BV/TV) were calculated over the entire vertebrae. The vertebral bodies were then mechanically tested to failure in compression, to determine ultimate load.

The variables $\mathrm{BV}_{\text {tot }}, \mathrm{BV}$, and $\mathrm{BV} / \mathrm{TV}$ measured by $\mu \mathrm{CT}$ were better predicted by $\mathrm{BMC}$ and BMD measured by lateral-projection DXA, with higher $\mathrm{R}^{2}$ values and smaller standard errors of the estimate $\left(\mathrm{R}^{2}=0.65-0.90, \mathrm{SEE}=11 \%-18 \%\right)$, compared to PAprojection DXA $\left(\mathrm{R}^{2}=0.33-0.53, \mathrm{SEE}=22 \%-34 \%\right)$. The best predictors of ultimate load were $B V_{\text {tot }}$ and $B V$ assessed by $\mu C T\left(R^{2}=0.88\right.$ and $R^{2}=0.81$, respectively), and BMC and BMD from lateral-projection DXA $\left(\mathrm{R}^{2}=0.82\right.$ and $\mathrm{R}^{2}=0.70$, respectively). Conversely, BMC and BMD from PA-projection DXA were lower predictors of ultimate load $\left(\mathrm{R}^{2}=0.49\right.$ and $\mathrm{R}^{2}=0.37$, respectively).

This ex vivo study highlights greater capabilities of lateral-projection DXA to predict variations in vertebral body bone quantity as measured by $\mu \mathrm{CT}$, and to predict vertebral strength as assessed experimentally, compared to PA-projection DXA. This provides basis for further exploring the clinical application of lateral-projection DXA analysis.

Keywords: vertebra, osteoporosis, densitometry, DXA, micro-CT, mechanical strength 


\section{$\underline{\text { Introduction }}$}

Dual energy X-ray absorptiometry (DXA) is currently the clinical tool of first choice for measuring areal bone mineral density (BMD) and for making clinical decisions concerning vertebral fragility and response to therapies. This is mainly due to its high precision, accuracy, efficiency, low radiation dose, accessible measurement sites, and low cost relative to other densitometry techniques [1-3]. However, marked differences in the prevalence of vertebral fractures for a comparable BMD, as measured by DXA, are reported [4-11]. Possible explanations for these discrepancies include the inability to measure three-dimensional (3D) bone microstructure and bone quality, confounding factors such as degeneration and sclerosis, extra-skeletal calcium, inadequate measurement specificity, the generally stochastic nature of vertebral fractures and the influence of clinical risk factors for fracture other than BMD [11].

For the clinical measurement of vertebral BMD, routinely posterior-anterior (PA) projections are taken of the spine with the patient lying supine on the scanner bed. However, in this scanning modality, the projection data also include the posterior vertebral elements, and they also potentially include aortic calcifications and degenerative spinal conditions in some patients. This can influence, particularly overestimate, the measured bone mineral content (BMC) and BMD data to an unknown extent, constituting an unwanted bias $[12,13]$. However, these bias elements can be excluded or minimized with a lateral projection scanning approach $[7,14]$. In a number of studies, BMD estimated by lateral-projection DXA has shown stronger relationships with vertebral fractures and age, compared to BMD using PA-projection DXA [14-16]. 
Moreover, the treatment effects of a pharmacologic intervention were greater when evaluated by lateral BMD data, in contrast to PA-derived data [17,18].

The ability of bone as an organ to withstand an applied load is also linked to its internal microarchitecture. BMD measurements made using DXA are derived from planar projections, from which no distinction between cortical and trabecular bone can be made. Vertebral bodies have trabecular bone structures with thicknesses as small as $100 \mu \mathrm{m}$ $[19,20]$, hence, 3D imaging methods with high resolution are essential for their accurate description. The development of high-resolution 3D imaging techniques, such as microcomputed tomography $(\mu \mathrm{CT})$ has made it possible to assess trabecular bone structure in 3D non-destructively [21]. The first studies characterizing vertebral microarchitecture with $\mu \mathrm{CT}$ were based on excised bone cores $[19,22]$. In order to then study mechanical properties, the cores were subjected to mechanical testing [22-24]. It is now possible to scan the whole vertebral body, rather than an excised core [25-29]. This provides highresolution structural data of the whole vertebra in $3 \mathrm{D}$, which can be used in combination with finite element models [26], or with mechanical test data of the whole vertebral body [20,30,31], to examine the resistance to fracture of the vertebra from which vertebral fragility can be inferred.

Accurate assessment of vertebral strength is of clinical importance for the management of bone fragility, and DXA is currently the clinical tool most widely used to inform clinical decisions regarding fracture risk. In vitro studies, examining BMC and BMD measurements on lumbar vertebrae from lateral-projection DXA compared to PA- 
projection DXA, suggested a more accurate prediction of lumbar vertebral failure load for lateral-projection DXA, with higher coefficients of determination and lower standard errors of the estimate in regression models [32,33]. Lateral-projection DXA might have some potential advantages for assessing vertebral bone status compared to PA-projection DXA, in particular as lateral scans might better approximate the objective of measuring the trabecular-rich bone in the vertebral body $[14,17]$. As such, it is valuable to investigate the capability of lateral-projection DXA, compared to PA-projection DXA, in predicting variations of bone quantity in human vertebrae as assessed by a highresolution 3D imaging modality such as $\mu \mathrm{CT}$.

The aims of this study on excised human spines were (1) to investigate the ability of bone quantity measurements (BMC and BMD) obtained by DXA in the lateral projection and in the PA projection, to predict variations in bone quantity measurements (bone volume and bone volume fraction) obtained by $\mu \mathrm{CT}$, (2) to assess the capability of the bone quantity measurements obtained by DXA in both the lateral and the PA projections, and of the bone quantity measurements obtained by $\mu \mathrm{CT}$, to predict vertebral body failure strength determined experimentally. In particular this study will investigate (1) whether vertebral bone quantity assessed by lateral-projection DXA, compared to PA-projection DXA, will better predict variations in bone quantity as determined by $\mu \mathrm{CT}$, and (2) whether vertebral bone quantity assessed from lateral-projection DXA, compared to PAprojection DXA, will better predict vertebral failure load. 


\section{$\underline{\text { Materials and Methods }}$}

\section{Study design}

This multi-centre project is currently run through three Australian centres [25,34]: Curtin University, Western Australia; SA Pathology, South Australia; and University of Melbourne (Royal Melbourne Hospital), Victoria. Initially, DXA scanning was performed in Melbourne, followed by $\mu \mathrm{CT}$ scanning and biomechanical testing in Adelaide. Finally data were analysed at both these sites and in Perth.

\section{Specimens}

Lumbar spine specimens (L2, L3) from eight embalmed cadavers (5 male, 3 female) of mean (SD) age at death 77.5 (10.4) years were used in this investigation. The intact cadavers were embalmed with 20-40L of embalming fluid (55\% ethanol, 5\% formaldehyde, $5 \%$ phenol, $20 \%$ propylene glycol and $15 \%$ water) and stored at $4{ }^{\circ} \mathrm{C}$ for 3 months prior to harvesting of the spine. Previous investigators have found no effect of formalin fixation on BMD estimations by DXA on cadaver spines compared to fresh specimens, over this timescale $[35,36]$. The ribs and ilia were removed, leaving intact spine segments with vertebrae, discs and posterior vertebral elements in place, from T12L5. The spines were sealed in a water-tight shrink-wrap thermoplastic. An experienced medical scientist examined the spines to identify vertebral levels ex situ. Prior to any scanning, lateral radiographs were acquired from each specimen to screen for vertebral fractures and any other obvious pathology and to verify vertebral levels in conjunction with the postero-anterior (PA) DXA image. One L2 vertebra showed a fracture and thus was excluded from the analyses, whereas the L3 vertebra of the same spine was 
maintained. All samples were free of any serological conditions. Approval to use the specimens for research purposes was granted by the Human Research Ethics Committee at the Royal Adelaide Hospital, South Australia, and Curtin University, Western Australia, in accordance with the Declaration of Helsinki, 1975. The resulting 15 L2 and L3 vertebrae ( $n=7$ and $n=8$, respectively) were used for both quantitative bone imaging modalities. The scanning and analysis procedures have been described in detail elsewhere using a protocol developed by our group [25,34,37,38], and are outlined below.

\section{Dual Energy X-ray Absorptiometry (DXA)}

All scanning was performed using a Hologic (Hologic Inc., Bedford, MA; USA) QDR4500A fan beam densitometer, running operating software version 9.10D. The 12 month precision of the densitometer for the Hologic spine phantom was $0.39 \%$ for BMD and $0.58 \%$ for BMC. Spines samples were placed supine in a water bath $(270 \times 180 \times 150 \mathrm{~mm})$ of tap water to a depth of $18 \mathrm{~cm}$ to simulate soft tissue composition. Specimens were wrapped in water-tight plastic wrap free of air and were secured to the base of the water bath with Velcro straps to prevent motion and rotation artefact during scanning. This procedure has been used in previous studies with validity and reliability established for both lumbar and thoracic vertebrae [33,37,39-41]. A matched PA-supine lateral scan was performed on each specimen using the array scanning mode. Analysis of the PA scan was performed according to the standard lumbar spine protocol described by Hologic (Fig. 1a) [42]. At the completion of the lateral scan, standard analysis was performed. To ensure standardisation, the global region of interest width remained constant throughout the analysis, set at its maximum $(141 \times 152$ pixels (pixel size $=$ 
1.0mm). Areal BMD was calculated for the whole vertebral body area (defined as region of interest, ROI) (Fig. 1b). The whole vertebral area (ROI) was defined by the four corners of the vertebra of interest from the lateral DXA image, including the vertebral endplate and excluding the posterior elements, as described previously [25,34,38]. Overt osteophytes were excluded from the ROI and deleted from the bone map manually, in agreement with previous work $[25,40,43,44]$. The size and shape of the ROI was defined according to the morphology of the vertebral body. The endplates defined the superior and inferior margins, the anterior border of the centrum defined the anterior margin, while the posterior margin was defined by the junction between the vertebral centrum and pedicle of the posterior elements. In circumstances where the automated process of bone map creation failed to detect bone tissue adequately, the map was completed manually. Measurement of lateral BMD and BMC using this protocol has been performed previously, both in vivo [38] and ex vivo [37] with good precision established. An earlier study established short-term intra-rater precision for this method by scanning 6 cadaver spines twice, one week apart, with an ICC (95\% CI) of $0.98(0.90-1.00)$ and $0.99(0.95-$ 1.00) at L2 and L3 [45], respectively.

\section{2. $\quad$ Micro-Computed Tomography $(\mu C T)$}

For $\mu \mathrm{CT}$ examination, the L2 and L3 vertebrae were individually dissected from the spine, and the posterior elements removed from each isolated vertebral body, by means of a bandsaw. $\mu \mathrm{CT}$ examination of each vertebra was performed using a desktop $\mu \mathrm{CT}$ system (Skyscan 1076, in vivo $\mu \mathrm{CT}$ scanner, Skyscan NV, Kontich, Belgium), as described previously [25]. The scan was done with the vertebral body fixed on a carbon 
bed, having the specimen axis in common with the rotation axis of the system. Scans were performed with a source voltage $80 \mathrm{kVp}$, current $120 \mu \mathrm{A}$, rotation step $0.5^{\circ}$, and full rotation over $180^{\circ}$, and a $0.5 \mathrm{~mm}$-thick aluminium filter for beam hardening reduction. The pixel size was $17.4 \mu \mathrm{m}$, exposure time 0.59 seconds, 4 frames averaging. Each scan was performed in three consecutive, automated steps, imaging a third of the specimen's height at each scan step. This produced a total of 1266 projection images (422 projections at each scan step), image size $1048 \times 3936$ pixels (length $\times$ side). The cross-section images were then reconstructed using a filtered back-projection algorithm (NRecon software, V 1.4.4, Skyscan, Kontich, Belgium). For each vertebra, a stack of up to 2500 crosssections was reconstructed, with an inter-slice distance of 1 pixel $(17.4 \mu \mathrm{m})$, corresponding to a maximum reconstructed height of $44 \mathrm{~mm}$ (Fig. 2), recreating the full height of the L2 and L3 vertebra. The reconstructed axial cross-section images were of $3936 \times 3936$ pixels each, $17.4 \mu \mathrm{m}$ pixel size, corresponding to images of $68.5 \times 68.5 \mathrm{~mm}$.

The volume scanned by $\mu \mathrm{CT}$ comprised the entire vertebral body. From the stack of contiguous cross-section images, a volume of interest (VOI) containing only trabecular bone was extracted, for calculation of the trabecular bone volume (BV) and trabecular bone volume fraction (BV/TV) (software CT Analyser V 1.8.0.5, Skyscan, Kontich, Belgium). The trabecular VOI started at a distance of $1 \mathrm{~mm}$ from both endplates and extended through the whole vertebra, corresponding to a height of $20.8 \pm 1.2 \mathrm{~mm}$ on average (mean $\pm \mathrm{SD}$ ) (Fig. 2). Over the same height, the total bone volume $\left(\mathrm{BV}_{\text {tot }}\right)$ was calculated, which included both the cortical and the trabecular bone. For calculation of $\mathrm{BV}_{\text {tot }}, \mathrm{BV}$, and $\mathrm{BV} / \mathrm{TV}$, the cross-section images were segmented (thresholded) into bone 
and non-bone using a uniform threshold algorithm, with a single threshold value applied to all specimens. The total bone volume $\left(\mathrm{BV}_{\text {tot, }}\right.$ expressed in $\left.\mathrm{mm}^{3}\right)$ was calculated as the volume occupied by the voxels segmented as bone (=cortical + trabecular); the trabecular bone volume $\left(\mathrm{BV}\right.$, expressed in $\mathrm{mm}^{3}$ ) was calculated as the volume occupied by the voxels segmented as bone within the trabecular VOI; the trabecular bone volume fraction (BV/TV, expressed in \%) was calculated as the voxels segmented as bone within the trabecular VOI divided by the voxels constituting the examined trabecular VOI, using the marching cubes method $[46,47]$. The average cross-sectional area of the vertebra (CSA, measured in $\mathrm{mm}^{2}$ ) was calculated, by computing the area enclosed by the cortex in each cross-section image, and averaging it over the height of the VOI.

\section{Compressive testing}

After $\mu \mathrm{CT}$ scanning, the vertebral bodies were mechanically tested to failure, to characterize their compressive strength. The specimen preparation and testing procedure was performed based on previously published protocols [20,48,49]. A 3mm-thick layer of polymethylmethacrylate (PMMA) was applied on both the endplates, by submerging the specimen into a curing PMMA mixture. The specimens were then soaked for $12 \mathrm{hrs}$ in saline solution for rehydration [33], before being placed in the mechanical testing machine (Model 800L, TestResources Inc, Shakopee MN, USA). Uniaxial compression tests were performed between steel platens with a lockable ball joint to ensure planoparallel ends $[48,50]$. The specimens were kept moist during the experiment with the use of saline-soaked gauze. After preconditioning $(5$ cycles, at $0.1 \mathrm{~Hz}$, between $150 \mathrm{~N}$ and $350 \mathrm{~N}$, then held at $250 \mathrm{~N}$ for 5 minutes), the specimens were tested in displacement 
control at a rate of $0.15 \mathrm{~mm} / \mathrm{s}$ at room temperature $[20,48]$. The displacement was measured by a transducer (Linear Variable Differential Transformer) attached to the cross-head. Vertebral body strength $\left(\mathrm{F}_{\mathrm{ult}}\right.$, expressed in $\left.\mathrm{kN}\right)$ was defined as the peak load during the loading cycle (ultimate load).

\section{$\underline{\text { Statistical analysis }}$}

Univariate linear regression analysis was used to determine the ability of DXA measurements (BMC, BMD) in both the lateral and the PA projection, to predict $\mu \mathrm{CT}$ measurements $\left(\mathrm{BV}_{\mathrm{tot}}, \mathrm{BV}, \mathrm{BV} / \mathrm{TV}\right)$ (SAS software, SAS Inc, Cary, NC, USA). Regression analysis was also used to determine the ability of BMC and BMD measured by DXA in both the projection modalities, as well of $\mathrm{BV}_{\mathrm{tot}}, \mathrm{BV}$ and $\mathrm{BV} / \mathrm{TV}$ measured by $\mu \mathrm{CT}$, to predict ultimate load. The difference in $\mathrm{R}^{2}$ values between the various regressions was assessed using Steiger's $Z$ test for dependent samples [51]. The significance level for all the tests was set at $\mathrm{p}=0.05$.

Some of the examined parameters (eg, BMC, BV, $\mathrm{F}_{\mathrm{ult}}$ ) can be bone size-dependent [20]. To investigate this dependency, univariate linear regression analysis was performed between all the examined parameters and the average cross-sectional area of the vertebra (CSA) obtained by $\mu \mathrm{CT}$. Furthermore, stepwise forward regression analysis was performed, to investigate whether in a linear model the inclusion of CSA as an additional independent variable to bone quantity would improve the prediction of ultimate load as an independent factor. 


\section{$\underline{\text { Results }}$}

All the cadaver spines were successfully scanned ex situ by DXA in PA projection and lateral projection, the corresponding L2 and L3 vertebrae dissected and scanned by $\mu \mathrm{CT}$, and then mechanically tested to failure. Descriptive data derived from the DXA analysis, $\mu \mathrm{CT}$ analysis, and mechanical testing, are summarized in Table 1 . The coefficients of determination of the various regressions are summarized in Tables 2-4. A threedimensional $\mu \mathrm{CT}$ representation of a vertebral body and of the trabecular bone compartment is shown in Fig. 3.

$\mu C T$ vs. DXA:

The regression models between bone quantity measurements from $\mu \mathrm{CT}$ and those from DXA were statistically significant $(\mathrm{p}<0.05$ in all cases). The variables measured by $\mu \mathrm{CT}$ were better predicted by variables estimated from lateral-projection DXA compared to PA-projection DXA, as indicated by higher $\mathrm{R}^{2}$ values and smaller standard errors of the estimate (SEE, expressed as \% of the average value) (Table 2). The coefficient of determination for "BV tot vs. BMC lateral-projection $\mathrm{DXA}$ " $\left(\mathrm{R}^{2}=0.90\right)$ was significantly higher $(\mathrm{p}<0.01)$ than for "BV tot vs. BMC PA-projection DXA" $\left(\mathrm{R}^{2}=0.53\right)$ (Fig. 4a and Fig. 4b, respectively). Similarly, the coefficient of determination for "BV vs. BMC lateral-projection DXA" $\left(\mathrm{R}^{2}=0.82\right)$ was significantly higher $(\mathrm{p}<0.01)$ than for "BV vs. BMC PA-projection DXA" $\left(\mathrm{R}^{2}=0.33\right)$ (Fig. 4c and Fig. 4d, respectively). The coefficient of determination for "BV/TV vs. BMD lateral-projection DXA" $\left(\mathrm{R}^{2}=0.65\right)$, was higher compared to "BV/TV vs. BMD PA-projection DXA" $\left(\mathrm{R}^{2}=0.41\right)$, although the difference did not reach statistical significance ( $\mathrm{p}=0.12$ ) (Fig. 4e and Fig. 4f, respectively). 
Ultimate load vs. bone quantity:

The regression models between ultimate load and the bone quantity measurements from DXA were significant $(\mathrm{p}<0.01$ in all cases). Ultimate load was better predicted by data from lateral-projection DXA compared to prediction by data from PA-projection DXA, showing higher $\mathrm{R}^{2}$ and lower SEE values.

The coefficient of determination for " $F_{u l t}$ vs. BMC lateral-projection DXA" $\left(\mathrm{R}^{2}=0.82\right)$, compared to "Fult vs. BMC PA-projection DXA" $\left(\mathrm{R}^{2}=0.49\right)$, was higher, with increase close to significance ( $\mathrm{p}=0.051$ ) (Fig. 5a and Fig. 5b, respectively). The coefficient of determination for " $F_{\text {ult }}$ vs. BMD lateral-projection DXA" $\left(\mathrm{R}^{2}=0.70\right)$, compared to " $F_{\text {ult }}$ vs. BMD PA-projection DXA” $\left(\mathrm{R}^{2}=0.37\right)$, was also higher, with statistically significant increase (p<0.05) (Fig. 5c and Fig. 5d, respectively).

The linear regressions between ultimate load and the bone quantity measurements from $\mu \mathrm{CT}$ were also significant ( $\mathrm{p}<0.01$ in all cases). The coefficient of determination for " $\mathrm{F}_{\text {ult }}$ vs. $\mathrm{BV}_{\text {tot" }}\left(\mathrm{R}^{2}=0.88\right)$ was higher compared to " $\mathrm{F}_{\text {ult }} \mathrm{vs.} \mathrm{BV}$ " $\left(\mathrm{R}^{2}=0.81\right)$, although the increase was not statistically significant ( $\mathrm{p}=0.21$ ) (Fig. 6a and Fig. 6b, respectively). Both of these coefficients of determination were higher than the coefficient of determination for "F $F_{\text {ult }}$ vs. BV/TV" $\left(\mathrm{R}^{2}=0.52\right)$ (Fig. 6c), with statistically significant increases $(\mathrm{p}<0.05$ for both). 
Dependency on CSA, and stepwise forward regression analysis:

Significant linear relationships with CSA $\left(\mathrm{R}^{2}=0.44-0.50, \mathrm{p}<0.01\right)$ were found for the parameters BMC lateral-projection DXA, $\mathrm{BV}$ and $\mathrm{BV}_{\text {tot }}$, as well as for $\mathrm{F}_{\text {ult }}$ (Table 3). In contrast, the parameters BMC PA-projection DXA, BMD lateral-projection DXA, BMD PA-projection DXA, and BV/TV, were not significantly related to CSA.

Stepwise forward regression analysis, with dependent variable $F_{u l t}$ and the two independent variables bone quantity and CSA, showed an increase in $\mathrm{R}^{2}$ for " $\mathrm{F}_{\text {ult }}$ vs. BMC PA-projection DXA + CSA", "Fult vs. BMD lateral-projection DXA + CSA", "Fult vs. BMD PA-projection DXA + CSA", and "F $F_{\text {ult }}$ vs. BV/TV + CSA" (Table 4). For the parameters $\mathrm{BMC}$ lateral-projection $\mathrm{DXA}, \mathrm{BV}_{\text {tot }}$, and $\mathrm{BV}$, adding the variable $\mathrm{CSA}$ in the analysis did not increase the prediction of $\mathrm{F}_{\mathrm{ult}}$ beyond the single regression model (Table 2) and were taken out of the analysis (Table 4).

\section{$\underline{\text { Discussion }}$}

In this study, human lumbar cadaver spines were examined ex situ by DXA in lateral- and PA projections as well as by $\mu \mathrm{CT}$ to determine bone quantity, and subsequently mechanically tested to determine vertebral body strength. DXA data derived from lateral projections demonstrated significantly stronger relationships with data obtained from high resolution $\mu \mathrm{CT}$ and vertebral strength than PA-derived DXA data, highlighting the potential advantages of lateral-projection DXA. 
$\mu C T$ vs. DXA

The $\mu \mathrm{CT}$-derived parameters $\mathrm{BV}_{\text {tot }}, \mathrm{BV}$ and $\mathrm{BV} / \mathrm{TV}$ were better predicted by the $\mathrm{BMC}$ and the BMD from lateral-projection DXA, in contrast to PA-projection DXA. This suggests that vertebral $\mathrm{BMC}$ and $\mathrm{BMD}$ measured using lateral-projection DXA are more strongly related to the vertebral body bone volume and trabecular bone volume fraction, respectively, compared to PA-projection DXA. The stronger relationships could be explained, at least in part, by the vertebral posterior elements not being included in the lateral-projection DXA scans, and thus lateral-projection DXA more closely measuring bone variations in the vertebral body as determined by $\mu \mathrm{CT}$, compared to PA-projection DXA. These ex vivo findings indicate that lateral-projection DXA has a greater sensitivity to detect bone quantity variations in the vertebral body than PA-projection DXA; hence, in principle, this suggests that lateral-projection DXA has a better ability to detect vertebral body bone changes, and as such the potential of greater diagnostic sensitivity, supporting previous in vivo findings in the literature $[17,18]$.

\section{Ultimate load vs. bone quantity}

The $\mathrm{BMC}$ and $\mathrm{BMD}$ measurements derived from lateral-projection DXA were strong predictors of ultimate load, highlighting the capability of lateral-projection DXA to predict vertebral body strength, with high coefficients of determination $\left(\mathrm{R}^{2}=0.82\right.$ and $\mathrm{R}^{2}=0.70$, respectively). This is in contrast to the commonly used PA-projection modality, which, although statistically significant, showed lower coefficients of determination for predicting ultimate load in this study $\left(R^{2}=0.49\right.$ and $R^{2}=0.37$, respectively $)$. This suggests that lateral-projection DXA scanning more closely measures bone variations in that part 
of the vertebra which is load bearing during uniaxial mechanical testing to failure, compared to PA-DXA scanning. This finding is in agreement with previous studies on human lumbar spines examined ex situ [32,33].

\section{Potential clinical implications}

This study shows improved predictions of variations in both the vertebral body bone quantity $\left(\mathrm{BV}_{\text {tot }}, \mathrm{BV}\right.$ and $\left.\mathrm{BV} / \mathrm{TV}\right)$ assessed by high-resolution $\mu \mathrm{CT}$, and in the failure load assessed experimentally, by using lateral-projection DXA, compared to PA-projection DXA. These data highlight potentially important clinical applications of lateral-projection DXA and the potential advantages of using lateral-projection approaches over PA projections. Further, these findings support those of previous studies for lateral-projection DXA, and highlight the underlying mechanism explaining the different detection capability, related to the different projection modalities in DXA.

Lateral-projection DXA was used only briefly when it was introduced 15-20 years ago [52-55]. This short window of clinical application was largely due to unacceptable precision associated with the approach, largely attributable to the requirement to reposition patients on the scanning table (e.g. from supine to decubitus lateral)[15]. For example, precision errors (expressed as \% CV) have ranged from 2.0-6.9\% with decubitus lateral approaches [56], and reduced considerably to clinically acceptable ranges with the introduction in the mid-1990's of supine-lateral approaches using rotating C-arm technology (e.g \% CV range 0.9-3.3\%) [7]. The rotating C-arm technology removes the need to reposition the patient for a lateral-projection scan, and enables the estimation of 
apparent volumetric BMD using both PA and lateral-projection data [15,32]. Further, matched supine-lateral scans enable baseline compensation algorithms to be applied to account for the effects of variable thickness and composition of soft tissue in the trunk in prospective scanning [56]. As further evidence emerges to substantiate the clinical benefits of acquiring lateral-projection images of the lumbar spine, more densitometers may be equipped with the rotating C-arm technology. However, it will be important to undertake prospective studies to determine the predictive strength of lateral-projection data, compared to PA-projection data, for vertebral fracture risk, before the true clinical benefit can be judged. While precision of the lateral-projection DXA BMD protocol used in this study is reported to be acceptable for ex vivo applications [37], precision for in vivo applications is likely to be more variable. The in vivo measurement variability with lateral-projection DXA is due to factors including the heterogeneous nature of soft tissue surrounding the lumbar spine, the variability of in vivo patient positioning, and the reduced photon fluxes with lateral projection because of greater soft tissue volume X-ray absorption. Nonetheless, in a sample of 5 individuals (mean age 60 years, range 54-64 years) the $\% \mathrm{CV}$ was $2.49 \%$ in the ROI placed in the whole vertebral body, in an investigation of short-term in vivo precision for the same DXA protocol [38], providing some evidence that the protocol is also sufficiently robust for in vivo applications. Precision of the DXA protocol for ex vivo applications may also vary according to the size of the cadaver specimens used. Future work should consider examining precision according to cadaver size, for example by gender. 
$\mu \mathrm{CT}$ is the non-destructive imaging modality capable of accurate micro-architectural examination of bone segments as big as excised human vertebral bodies (up to about 60$70 \mathrm{~mm}$ in diameter) with high spatial resolution in $3 \mathrm{D}$ (eg, $17.4 \mu \mathrm{m}$ voxel size) [25], and for which accurate discrimination between the trabecular bone compartment and remaining tissue is possible. Endplates likely derive their ultimate strength from the underlying trabeculae $[20,28]$, and, as found in the present study, trabecular parameters $\mathrm{BV}$ and BV/TV were strong predictors of vertebral body strength. A number of studies have investigated the contribution of the trabecular bone and the cortical shell to whole vertebral strength, however, differences among studies exist in quantifying this relative contribution, which might be also related to different experimental procedures [20,26,31,57-63]. In a recent ex vivo study with mechanical testing procedures similar to the present study, using multiple regression analysis, it was shown that cortical morphology was not significantly related to vertebral strength after accounting for trabecular microarchitecture [20]. Similarly, in another recent study, failure load as described by BMD in lateral-projection DXA showed an increase in the coefficient of determination in a multiple regression analysis when trabecular micro-architectural parameters were included, rather than when cortical parameters were included [31]. In the present study, the coefficient of determination for " $F_{u l t} v s . ~ B V_{\text {tot }}$ " $\left(R^{2}=0.88\right)$ was slightly increased compared to the (already high) coefficient of determination for " $F_{\text {ult }}$ Vs. BV" $\left(\mathrm{R}^{2}=0.81\right)$, yet this increase $(+7 \%)$ was not statistically significant $(\mathrm{p}=0.21)$. Hence, by adding the bone volume of the cortex to the bone volume of the trabeculae, the prediction of variations in failure load increased by a relatively small amount. Thus, despite acknowledging the role of cortical bone in influencing vertebral biomechanical 
properties, in particular regarding stiffness and work to failure [31,64], which was not examined in the present study, the trabecular bone appears to play the primary role in determining vertebral body strength, consistent with earlier data $[20,28,60]$.

The present findings, of the bone volume $\left(\mathrm{BV}_{\text {tot }}\right.$ and $\left.\mathrm{BV}\right)$ assessed by $\mu \mathrm{CT}$ as being a better predictor of vertebral failure load than the $\mathrm{BV} / \mathrm{TV}$, as well as the $\mathrm{BMC}$ assessed by DXA being a better predictor of vertebral failure load than the BMD, are consistent with previous studies on entire vertebrae $[20,33,65]$. This could be expected, since the BMC (and $\mathrm{BV}_{\text {tot }}$ and $\mathrm{BV}$ ) is more related to bone size than is the $\mathrm{BMD}$ (and BV/TV) (Table 3) [66-69], and failure loads do scale significantly with bone size [33,69] (Table 3). BMC measurements examine the whole bone specimen, reaffirming that the amount of bone tissue is strongly related to its load-bearing capacity [65].

Stepwise forward regression analysis showed that for bone quantity parameters assessed by DXA there was an increase in the prediction of ultimate load if CSA is included in the analysis, such as "Fult vs. BMC PA-projection DXA + CSA", "Fult vs. BMD lateralprojection DXA + CSA", and "Fult vs. BMD PA-projection DXA + CSA" (Table 4), similar to previously reported results by others [28]. However, despite this increase when combined with CSA, the BMC and BMD measured by using lateral projection-DXA still showed higher coefficients of determination in the regression with failure load compared to PA-projection DXA, confirming the findings of the univariate regression analysis (Table 2). 
An additional analysis was also done, with the regressions for "bone strength vs. bone quantity" examined in terms of "ultimate stress vs. bone quantity", for which ultimate stress (measured in $\mathrm{MPa}$ ) was calculated as "ultimate stress $=\mathrm{F}_{\mathrm{ult}} / \mathrm{CSA}$ " (data not shown). The main findings for ultimate stress were the same as those reported for ultimate load in the manuscript, with BMC and BMD from lateral-projection DXA being better predictors of stress than from PA-projection DXA. Moreover, apart from the regression "ultimate stress vs. BMD PA-projection DXA" $\left(\mathrm{R}^{2}=0.52\right)$ and "ultimate stress vs. BV/TV" $\left(\mathrm{R}^{2}=0.73\right)$, in all the other examined regressions "ultimate stress vs. bone quantity" (BV, $\mathrm{BV}_{\text {tot }}$, BMC lateral-projection DXA, BMC PA-projection DXA, BMD lateral-projection DXA), the coefficients of determination for ultimate stress were lower compared to those for ultimate load, in agreement with previous findings on human vertebrae [70]. Thus, in this study, the focus in the regression analyses was on ultimate load, rather than ultimate stress.

Previous studies have investigated lateral-projection DXA and PA-projection DXA in the prediction of bone strength [32,33], other studies investigated lateral-projection DXA with $\mu \mathrm{CT}$ in the prediction of bone strength [31]. Further studies compared the commonly used PA-projection DXA with other clinical densitometric techniques, such as quantitative computed tomography (QCT), peripheral QCT, high-resolution peripheral QCT and ultrasound, in the prediction of bone strength [28,71]. However, to the authors' knowledge, no study has yet examined the capability of lateral-projection DXA compared to PA-projection DXA, to predict the variations in bone quantity measurements as described by a high-resolution 3D imaging modality such as $\mu \mathrm{CT}$, (which has the highest 
spatial resolution in 3D compared to the aforementioned imaging devices), and their respective capabilities to predict bone strength determined experimentally. The comparison of the two DXA-projection modalities in predicting variations in bone quantity with a high-resolution 3D reference modality such as $\mu \mathrm{CT}$, and their respective capabilities to predict bone strength determined experimentally, highlights the methodologic strength and novelty in this study.

A limitation of this study is the sample size (15 specimens in total), which nonetheless is comparable to that of other studies in this field, in particular using whole vertebral body $\mu \mathrm{CT}$ imaging combined with mechanical testing $(\mathrm{n}=16, \mathrm{n}=21, \mathrm{n}=16)[20,64,72]$. Another potential limitation in this study was the use of two vertebrae from each donor, thus data points were not truly independent. However, given that the primary aim was to explore the ability of bone quantity measurements obtained by the two DXA projection modalities to predict variations in bone measurements from $\mu \mathrm{CT}$, and their capability to predict failure load, for single vertebrae, and thereby utilising a repeated betweenvertebra design, we do not consider this to be a significant issue [34].

The specimens were obtained from cadavers with a relatively advanced age at death, and as such are more representative of an aged population subgroup, which might be considered relevant for risk of fracture, given the independent contribution of age to vertebral fragility. Indeed, the BMD values obtained in PA-projection DXA are lower compared to the PA-BMD values for the L2-4 spines of the young adult reference population in Australia (Geelong Osteoporosis Study [73,74]). However, a straight 
forward comparison is inappropriate in this context, as the reference values for the healthy population are measured in situ and in women, whereas in our study we have values measured ex situ, including both men and women. For men, a comparison with reference values is making an improper conversion for Lunar-scanner (Lunar Corporation, Madison, WI) to Hologic-scanner derived BMD values, as the formulae for the conversion between scanner types were obtained for women and not for men [73-75]. Moreover, Lochmueller et al., although using a DXA scanner of a different manufacturer (Lunar), showed that ex situ values overestimate in situ values by 10\% BMD [36]. However, with the necessary caution, in converting the here reported values into in situ values [36], and applying the conversion between scanner types (Lunar vs. Hologic) [7375], both females $\left(0.657 \pm 0.186 \mathrm{~g} / \mathrm{cm}^{2}\right)$ and males $\left(0.789 \pm 0.159 \mathrm{~g} / \mathrm{cm}^{2}\right)$ fell into the osteoporotic range (average values below $-2.5 \mathrm{SD}$ ). This, together with the advanced age, suggests that subjects sampled in our study might generally represent a subgroup with an increased risk of fracture compared to the normal population.

A further limitation is that the present study examined human spines ex situ immersed in a water bath. As such it is not taking into account the in vivo problems related to different amounts of soft tissues for different patients, thus representing a best case scenario for the measurement of BMD, and for predicting failure load. However, in a previously published study, lumbar spines were examined by DXA in situ on cadavers, and then $e x$ situ with excised spines immersed in a water bath, in PA projection and also in lateral projection, and mechanically tested [33]. According to the findings in that study, the lower correlation with failure load found for BMD measurements in PA-projection scans 
in comparison to the lateral-projection scans, appears to origin from the inclusion of the posterior elements in the DXA measurement, rather than from soft tissue errors or extraskeletal calcifications [33]. Nonetheless, as the present study examined excised spines immersed in a water bath, it is not fully representative of a true in vivo scan, with the variable amount of soft-tissue in place, which depends on the individual examined. As such, these results cannot be directly extrapolated into an in vivo scanning condition, and as mentioned earlier in this manuscript, further studies with densitometers using rotating C-arm technology are needed, to address accuracy and precision according to cadaver size and by gender [15,32].

The loading conditions for biomechanical tests were chosen to provide controlled boundary conditions common in laboratory testing, and might not be fully representative of in vivo loading conditions $[20,48]$. The focus in this study was on compressive testing, as functional loads in the lumbar spine are primarily compressive in nature [76], although it is acknowledged that loading conditions vary in other spinal levels where vertebral fractures are common [77]. For example, the uniaxial compressive test does not represent a loading condition during flexed posture which can give rise to wedge fractures, and for which in vivo loads are not well understood [78]. Further studies are needed to address this issue.

In conclusion, in this ex vivo study of lumbar vertebral bodies, high coefficients of determination in the regressions between bone quantity parameters measured from lateral-projection DXA (BMC and BMD), and bone quantity parameters determined from 
$\mu \mathrm{CT}\left(\mathrm{BV}_{\text {tot }}, \mathrm{BV}\right.$ and $\left.\mathrm{BV} / \mathrm{TV}\right)$ were found. It was shown that BMC and BMD measured using lateral-projection DXA, in contrast to PA-projection DXA, are strong predictors of the amount of bone in the vertebral body as measured by $\mu \mathrm{CT}$. Both BMC and BMD measurements in lateral-projection DXA were good predictors of ultimate load, highlighting the capability of using lateral-projection DXA to predict vertebral body strength. This is in contrast to the commonly used PA-projection DXA, which showed significantly lower coefficient of determination for predicting ultimate load. These findings support further exploration of the clinical application of lateral-projection DXA analysis in fracture prediction.

\section{Acknowledgements}

Funding for these studies was provided by the National Health and Medical Research Council (NHMRC) of Australia, Scoliosis Research Society (USA), and Arthritis Australia. Dr Andrew Briggs is supported by a fellowship awarded by the NHMRC. In kind support was provided by the University of Melbourne Department of Medicine (Royal Melbourne Hospital), and SA Pathology.

\section{References}

[1] Kleerekoper M, Nelson DA. Which bone density measurement? J Bone Miner Res 1997;12:712-4.

[2] Blake GM, Fogelman I. Role of dual-energy X-ray absorptiometry in the diagnosis and treatment of osteoporosis. J Clin Densitom 2007;10:102-10.

[3] Kanis JA, Burlet N, Cooper C, Delmas PD, Reginster JY, Borgstrom F, Rizzoli R. European guidance for the diagnosis and management of osteoporosis in postmenopausal women. Osteoporos Int 2008;19:399-428.

[4] Marshall D, Johnell O, Wedel H. Meta-analysis of how well measures of bone mineral density predict occurrence of osteoporotic fractures. Bmj 1996;312:12549. 
[5] McCreadie BR, Goldstein SA. Biomechanics of fracture: is bone mineral density sufficient to assess risk? J Bone Miner Res 2000;15:2305-8.

[6] Hordon LD, Raisi M, Aaron JE, Paxton SK, Beneton M, Kanis JA. Trabecular architecture in women and men of similar bone mass with and without vertebral fracture: I. Two-dimensional histology. Bone 2000;27:271-6.

[7] Jergas M, Breitenseher M, Gluer CC, Black D, Lang P, Grampp S, Engelke K, Genant HK. Which vertebrae should be assessed using lateral dual-energy X-ray absorptiometry of the lumbar spine. Osteoporos Int 1995;5:196-204.

[8] Legrand E, Chappard D, Pascaretti C, Duquenne M, Krebs S, Rohmer V, Basle MF, Audran M. Trabecular bone microarchitecture, bone mineral density, and vertebral fractures in male osteoporosis. J Bone Miner Res 2000;15:13-9.

[9] Peel NF, Moore DJ, Barrington NA, Bax DE, Eastell R. Risk of vertebral fracture and relationship to bone mineral density in steroid treated rheumatoid arthritis. Ann Rheum Dis 1995;54:801-6.

[10] Arabi A, Baddoura R, Awada H, Khoury N, Haddad S, Ayoub G, El-Hajj Fuleihan G. Discriminative ability of dual-energy X-ray absorptiometry site selection in identifying patients with osteoporotic fractures. Bone 2007;40:10605.

[11] Briggs AM, Greig AM, Wark JD. The vertebral fracture cascade in osteoporosis: a review of aetiopathogenesis. Osteoporos Int 2007;18:575-84.

[12] Drinka PJ, DeSmet AA, Bauwens SF, Rogot A. The effect of overlying calcification on lumbar bone densitometry. Calcif Tissue Int 1992;50:507-10.

[13] Yu W, Gluer CC, Fuerst T, Grampp S, Li J, Lu Y, Genant HK. Influence of degenerative joint disease on spinal bone mineral measurements in postmenopausal women. Calcif Tissue Int 1995;57:169-74.

[14] Zmuda JM, Cauley JA, Glynn NW, Finkelstein JS. Posterior-anterior and lateral dual-energy $\mathrm{X}$-ray absorptiometry for the assessment of vertebral osteoporosis and bone loss among older men. J Bone Miner Res 2000;15:1417-24.

[15] Duboeuf F, Pommet R, Meunier PJ, Delmas PD. Dual-energy X-ray absorptiometry of the spine in anteroposterior and lateral projections. Osteoporos Int 1994;4:110-6.

[16] Peel NF, Eastell R. Diagnostic value of estimated volumetric bone mineral density of the lumbar spine in osteoporosis. J Bone Miner Res 1994;9:317-20.

[17] Blake GM, Herd RJ, Fogelman I. A longitudinal study of supine lateral DXA of the lumbar spine: a comparison with posteroanterior spine, hip and total-body DXA. Osteoporos Int 1996;6:462-70.

[18] Reid IR, Evans MC, Stapleton J. Lateral spine densitometry is a more sensitive indicator of glucocorticoid-induced bone loss. J Bone Miner Res 1992;7:1221-5.

[19] Hildebrand T, Laib A, Müller R, Dequeker J, Rüegsegger P. Direct threedimensional morphometric analysis of human cancellous bone: microstructural data from spine, femur, iliac crest, and calcaneus. J Bone Miner Res 1999;14:1167-74.

[20] Fields AJ, Eswaran SK, Jekir MG, Keaveny TM. Role of trabecular microarchitecture in whole-vertebral body biomechanical behavior. J Bone Miner Res 2009;24:1523-30. 
[21] Müller R, Hahn M, Vogel M, Delling G, Rüegsegger P. Morphometric analysis of noninvasively assessed bone biopsies: comparison of high-resolution computed tomography and histologic sections. Bone 1996;18:215-20.

[22] Goulet RW, Goldstein SA, Ciarelli MJ, Kuhn JL, Brown MB, Feldkamp LA. The relationship between the structural and orthogonal compressive properties of trabecular bone. J Biomech 1994;27:375-89.

[23] Nazarian A, Stauber M, Zurakowski D, Snyder BD, Muller R. The interaction of microstructure and volume fraction in predicting failure in cancellous bone. Bone 2006;39:1196-202.

[24] Perilli E, Baleani M, Ohman C, Fognani R, Baruffaldi F, Viceconti M. Dependence of mechanical compressive strength on local variations in microarchitecture in cancellous bone of proximal human femur. $\mathrm{J}$ Biomech 2008;41:438-46.

[25] Briggs AM, Perilli E, Parkinson IH, Wrigley TV, Fazzalari NL, Kantor S, Wark JD. Novel assessment of subregional bone mineral density using DXA and pQCT and subregional microarchitecture using micro-CT in whole human vertebrae: applications, methods, and correspondence between technologies. J Clin Densitom 2010;13:161-74.

[26] Eswaran SK, Gupta A, Adams MF, Keaveny TM. Cortical and trabecular load sharing in the human vertebral body. J Bone Miner Res 2006;21:307-14.

[27] Homminga J, Van-Rietbergen B, Lochmuller EM, Weinans H, Eckstein F, Huiskes R. The osteoporotic vertebral structure is well adapted to the loads of daily life, but not to infrequent "error" loads. Bone 2004;34:510-6.

[28] Hulme PA, Boyd SK, Ferguson SJ. Regional variation in vertebral bone morphology and its contribution to vertebral fracture strength. Bone 2007;41:94657.

[29] Kim DG, Hunt CA, Zauel R, Fyhrie DP, Yeni YN. The effect of regional variations of the trabecular bone properties on the compressive strength of human vertebral bodies. Ann Biomed Eng 2007;35:1907-13.

[30] Wegrzyn J, Roux JP, Arlot ME, Boutroy S, Vilayphiou N, Guyen O, Delmas PD, Chapurlat R, Bouxsein ML. Role of trabecular microarchitecture and its heterogeneity parameters in the mechanical behavior of ex vivo human L3 vertebrae. J Bone Miner Res 2010;25:2324-31.

[31] Roux JP, Wegrzyn J, Arlot ME, Guyen O, Delmas PD, Chapurlat R, Bouxsein ML. Contribution of trabecular and cortical components to biomechanical behavior of human vertebrae: an ex vivo study. J Bone Miner Res 2010;25:35661.

[32] Myers BS, Arbogast KB, Lobaugh B, Harper KD, Richardson WJ, Drezner MK. Improved assessment of lumbar vertebral body strength using supine lateral dualenergy x-ray absorptiometry. J Bone Miner Res 1994;9:687-93.

[33] Burklein D, Lochmuller E, Kuhn V, Grimm J, Barkmann R, Muller R, Eckstein F. Correlation of thoracic and lumbar vertebral failure loads with in situ vs. ex situ dual energy X-ray absorptiometry. J Biomech 2001;34:579-87.

[34] Briggs AM, Perilli E, Parkinson IH, Kantor S, Wrigley TV, Fazzalari NL, Wark JD. Measurement of subregional vertebral bone mineral density in vitro using lateral projection dual energy X-ray absorptiometry (DXA): Validation with 
peripheral quantitative computed tomography (pQCT). J Bone Miner Metab 2011; in press, DOI:10.1007/s00774-011-0307-3.

[35] Edmondston SJ, Singer KP, Day RE, Breidahl PD, Price RI. Formalin fixation effects on vertebral bone density and failure mechanics: an in-vitro study of human and sheep vertebrae. Clin Biomech 1994;9:175-179.

[36] Lochmuller EM, Krefting N, Burklein D, Eckstein F. Effect of fixation, softtissues, and scan projection on bone mineral measurements with dual energy Xray absorptiometry (DXA). Calcif Tissue Int 2001;68:140-5.

[37] Briggs AM, Wark JD, Kantor S, Fazzalari NL, Greig AM, Bennell KL. Bone mineral density distribution in thoracic and lumbar vertebrae: an ex vivo study using dual energy X-ray absorptiometry. Bone 2006;38:286-8.

[38] Briggs AM, Wark JD, Kantor S, Teh R, Greig AM, Fazzalari NL, Bennell KL. In vivo intrarater and interrater precision of measuring apparent bone mineral density in vertebral subregions using supine lateral dual-energy x-ray absorptiometry. J Clin Densitom 2005;8:314-9.

[39] Singer K, Edmondston S, Day R, Breidahl P, Price R. Prediction of thoracic and lumbar vertebral body compressive strength: correlations with bone mineral density and vertebral region. Bone 1995;17:167-74.

[40] Edmondston SJ, Singer KP, Price RI, Breidahl PD. Accuracy of lateral dual energy X-ray absorptiometry for the determination of bone mineral content in the thoracic and lumbar spine: an in vitro study. Br J Radiol 1993;66:309-13.

[41] Kaymakci B, Wark JD. Precise accurate mineral measurements of excised sheep bones using X-ray densitometry. Bone Miner 1994;25:231-46.

[42] Hologic Inc. QDR 4500 X-ray Bone Densitometer User's Guide. Hologic Inc., Bedford, MA; USA. 1996.

[43] Sran MM, Khan KM, Keiver K, Chew JB, McKay HA, Oxland TR. Accuracy of DXA scanning of the thoracic spine: cadaveric studies comparing BMC, areal BMD and geometric estimates of volumetric BMD against ash weight and CT measures of bone volume. Eur Spine J 2005;14:971-6.

[44] Jones G, White C, Nguyen T, Sambrook PN, Kelly PJ, Eisman JA. Prevalent vertebral deformities: relationship to bone mineral density and spinal osteophytosis in elderly men and women. Osteoporos Int 1996;6:233-9.

[45] Briggs AM. Pathomechanics of spinal osteoporosis: Subregional bone mineral density and physiologic loading. PhD Thesis. School of Physiotherapy and Department of Medicine. Melbourne, The University of Melbourne. 2006b.

[46] Lane NE, Haupt D, Kimmel DB, Modin G, Kinney JH. Early estrogen replacement therapy reverses the rapid loss of trabecular bone volume and prevents further deterioration of connectivity in the rat. J Bone Miner Res 1999;14:206-14.

[47] Lorensen WE, Cline HE. Marching Cubes: A high resolution 3D surface construction algorithm. Computer Graphics 1987;21:163-169.

[48] Kopperdahl DL, Pearlman JL, Keaveny TM. Biomechanical consequences of an isolated overload on the human vertebral body. J Orthop Res 2000;18:685-90.

[49] Crawford RP, Cann CE, Keaveny TM. Finite element models predict in vitro vertebral body compressive strength better than quantitative computed tomography. Bone 2003;33:744-50. 
[50] Liebschner MA, Rosenberg WS, Keaveny TM. Effects of bone cement volume and distribution on vertebral stiffness after vertebroplasty. Spine (Phila Pa 1976) 2001;26:1547-54.

[51] Meng XL, Rosenthal R, Rubin DB. Comparing correlated correlation coefficients. Quantitative methods in psychology 1992;111:172-175.

[52] Bjarnason K, Hassager C, Svendsen OL, Stang H, Christiansen C. Anteroposterior and lateral spinal DXA for the assessment of vertebral body strength: comparison with hip and forearm measurement. Osteoporos Int 1996;6:37-42.

[53] Slosman DO, Rizzoli R, Donath A, Bonjour JP. Vertebral bone mineral density measured laterally by dual-energy X-ray absorptiometry. Osteoporos Int 1990;1:23-9.

[54] Mazess RB, Barden HS, Eberle RW, Denton MD. Age changes of spine density in posterior-anterior and lateral projections in normal women. Calcif Tissue Int 1995;56:201-5.

[55] Del Rio L, Pons F, Huguet M, Setoain FJ, Setoain J. Anteroposterior versus lateral bone mineral density of spine assessed by dual X-ray absorptiometry. Eur J Nucl Med 1995;22:407-12.

[56] Blake GM, Jagathesan T, Herd RJ, Fogelman I. Dual X-ray absorptiometry of the lumbar spine: the precision of paired anteroposterior/lateral studies. Br J Radiol 1994;67:624-30.

[57] Mosekilde L. Vertebral structure and strength in vivo and in vitro. Calcif Tissue Int 1993;53 Suppl 1:S121-5; discussion S125-6.

[58] Riggs BL, Melton LJ, 3rd. Involutional osteoporosis. N Engl J Med 1986;314:1676-86.

[59] Rockoff SD, Sweet E, Bleustein J. The relative contribution of trabecular and cortical bone to the strength of human lumbar vertebrae. Calcif Tissue Res 1969;3:163-75.

[60] Silva MJ, Keaveny TM, Hayes WC. Load sharing between the shell and centrum in the lumbar vertebral body. Spine (Phila Pa 1976) 1997;22:140-50.

[61] Andresen R, Werner HJ, Schober HC. Contribution of the cortical shell of vertebrae to mechanical behaviour of the lumbar vertebrae with implications for predicting fracture risk. Br J Radiol 1998;71:759-65.

[62] McBroom RJ, Hayes WC, Edwards WT, Goldberg RP, White AA, 3rd. Prediction of vertebral body compressive fracture using quantitative computed tomography. J Bone Joint Surg Am 1985;67:1206-14.

[63] Ebbesen EN, Thomsen JS, Beck-Nielsen H, Nepper-Rasmussen HJ, Mosekilde L. Lumbar vertebral body compressive strength evaluated by dual-energy X-ray absorptiometry, quantitative computed tomography, and ashing. Bone 1999;25:713-24.

[64] Wegrzyn J, Roux JP, Arlot ME, Boutroy S, Vilayphiou N, Guyen O, Delmas PD, Chapurlat R, Bouxsein ML. Determinants of the mechanical behavior of human lumbar vertebrae after simulated mild fracture. J Bone Miner Res 2011;26:73946. 
[65] Muller R, Hannan M, Smith SY, Bauss F. Intermittent ibandronate preserves bone quality and bone strength in the lumbar spine after 16 months of treatment in the ovariectomized cynomolgus monkey. J Bone Miner Res 2004;19:1787-96.

[66] Carter DR, Bouxsein ML, Marcus R. New approaches for interpreting projected bone densitometry data. J Bone Miner Res 1992;7:137-45.

[67] Prentice A, Parsons TJ, Cole TJ. Uncritical use of bone mineral density in absorptiometry may lead to size-related artifacts in the identification of bone mineral determinants. Am J Clin Nutr 1994;60:837-42.

[68] Jergas M, Breitenseher M, Gluer CC, Yu W, Genant HK. Estimates of volumetric bone density from projectional measurements improve the discriminatory capability of dual X-ray absorptiometry. J Bone Miner Res 1995b;10:1101-10.

[69] Lochmuller EM, Miller P, Burklein D, Wehr U, Rambeck W, Eckstein F. In situ femoral dual-energy X-ray absorptiometry related to ash weight, bone size and density, and its relationship with mechanical failure loads of the proximal femur. Osteoporos Int 2000;11:361-7.

[70] Edmondston SJ, Singer KP, Day RE, Price RI, Breidahl PD. Ex vivo estimation of thoracolumbar vertebral body compressive strength: the relative contributions of bone densitometry and vertebral morphometry. Osteoporos Int 1997;7:142-8.

[71] Lochmuller EM, Burklein D, Kuhn V, Glaser C, Muller R, Gluer CC, Eckstein F. Mechanical strength of the thoracolumbar spine in the elderly: prediction from in situ dual-energy X-ray absorptiometry, quantitative computed tomography (QCT), upper and lower limb peripheral QCT, and quantitative ultrasound. Bone 2002;31:77-84.

[72] Fields AJ, Lee GL, Liu XS, Jekir MG, Guo XE, Keaveny TM. Influence of vertical trabeculae on the compressive strength of the human vertebra. J Bone Miner Res 2011;26:263-9.

[73] Henry MJ, Pasco JA, Korn S, Gibson JE, Kotowicz MA, Nicholson GC. Bone mineral density reference ranges for Australian men: Geelong Osteoporosis Study. Osteoporos Int 2010;21:909-17.

[74] Henry MJ, Pasco JA, Pocock NA, Nicholson GC, Kotowicz MA. Reference ranges for bone densitometers adopted Australia-wide: Geelong osteoporosis study. Australas Radiol 2004;48:473-5.

[75] Genant HK, Grampp S, Gluer CC, Faulkner KG, Jergas M, Engelke K, Hagiwara S, Van Kuijk C. Universal standardization for dual x-ray absorptiometry: patient and phantom cross-calibration results. J Bone Miner Res 1994;9:1503-14.

[76] Adams MA, Dolan P. Spine biomechanics. J Biomech 2005;38:1972-83.

[77] Briggs AM, Wrigley TV, van Dieen JH, Phillips B, Lo SK, Greig AM, Bennell KL. The effect of osteoporotic vertebral fracture on predicted spinal loads in vivo. Eur Spine J 2006;15:1785-95.

[78] Crawford RP, Keaveny TM. Relationship between axial and bending behaviors of the human thoracolumbar vertebra. Spine (Phila Pa 1976) 2004;29:2248-55. 


\section{Figure legends}

Figure 1:

(a) DXA image of a lumbar spine taken in PA-projection. (b) Schematic image of a L2 vertebra, highlighting the region of interest used during lateral DXA scanning, defined by the 4 corners of the vertebral body in sagittal plane, which integrated cortical and trabecular bone (dashed line).

Figure 2:

Coronal and axial cross-section images of a vertebral body obtained from $\mu \mathrm{CT}$. The dashed line represents the trabecular bone compartment of the vertebra (voxel size $17 \mu \mathrm{m})$. Bone quantity by $\mu \mathrm{CT}$ was calculated as: total bone volume $\left(\mathrm{BV}_{\text {tot }}\right)$, which includes cortical and trabecular bone; trabecular bone volume (BV); and trabecular bone volume fraction (BV/TV).

Figure 3:

(a) 3D image of a vertebral body obtained by $\mu \mathrm{CT}$ (b) 3D image of the trabecular bone compartment of a vertebral body (voxel size $17 \mu \mathrm{m}$ ). Bone quantity by $\mu \mathrm{CT}$ was calculated as: total bone volume $\left(\mathrm{BV}_{\text {tot }}\right)$, which includes cortical and trabecular bone; trabecular bone volume (BV); and trabecular bone volume fraction (BV/TV).

Figure 4:

Scatter plots of bone quantity parameters determined by $\mu \mathrm{CT}\left(\mathrm{BV}_{\text {tot }}, \mathrm{BV}\right.$ and $\left.\mathrm{BV} / \mathrm{TV}\right)$, versus bone quantity parameters determined by DXA (BMC and BMD), in lateral 
projection and in PA projection. In the regressions, the determination coefficients for $\mathrm{BV}_{\text {tot }}, \mathrm{BV}$ and $\mathrm{BV} / \mathrm{TV}$ were higher with $\mathrm{BMC}$ and $\mathrm{BMD}$ in lateral projection DXA (Fig. 4a, 4c, 4e), compared to PA projection DXA (Fig. 4b, 4d, 4f). BMC measured in lateral projection DXA was highly predictive of $\mathrm{BV}_{\text {tot }}$ determined by $\mu \mathrm{CT}$ (Fig. $4 \mathrm{a}, \mathrm{R}^{2}=0.90$, $\mathrm{p}<0.0001$ ). $\mathrm{BV}_{\text {tot }}$, total bone volume (cortical and trabecular); $\mathrm{BV}$, trabecular bone volume; $\mathrm{BV} / \mathrm{TV}$, trabecular bone volume fraction.

\section{Figure 5:}

Scatter plots of ultimate load ( $\left.\mathrm{F}_{\text {ult }}\right)$ versus bone quantity parameters (BMC and BMD) determined by DXA, in lateral projection and in PA projection. In the regressions, the coefficients of determination for ultimate load were higher with BMC and BMD in lateral projection DXA (Fig. 5a and 5c), compared to PA projection DXA (Fig. 5b and 5d). BMC measured in lateral projection DXA was the best predictor of ultimate load $\left(\mathrm{R}^{2}=\right.$ $0.82, \mathrm{p}<0.0001$, Fig. 5a).

Figure 6:

Scatter plots of ultimate load $\left(\mathrm{F}_{\mathrm{ult}}\right)$ versus bone quantity parameters $\mathrm{BV}_{\text {tot }}, \mathrm{BV}$ and $\mathrm{BV} / \mathrm{TV}$ determined by $\mu \mathrm{CT}$ (Fig. $6 \mathrm{a}, 6 \mathrm{~b}, 6 \mathrm{c}$ ). $\mathrm{BV}_{\text {tot }}$ measured by $\mu \mathrm{CT}$ was best predictor of ultimate load $\left(\mathrm{R}^{2}=0.88, \mathrm{p}<0.0001\right)$. $\mathrm{BV}_{\text {tot }}$, total bone volume (cortical and trabecular); $\mathrm{BV}$, trabecular bone volume; $\mathrm{BV} / \mathrm{TV}$, trabecular bone volume fraction. 